\title{
WAVE ACTIVITY IN THE NEIGHBORHOOD OF THE BOWSHOCK OF MARS
}

\author{
R.Z. Sagdeev, V.D. Shapiro, V.I. Shevchenko, A. Zacharov,
}

P. Király, K. Szegó, A.F. Nagy, R.J.L. Grard

Ahstrac. Plasma wave activity in the neighborhood of the Martin bow shock were measured for the first time by the Sowiet spacecraft Phobos- 2 in a wide frequency range from dc $0150 \mathrm{kHz}$. The wave activity varied in character as the spucecraft moved across different plasma regions: in the seighborhood of the Martian bow shock, inside the magnetosheath and in the tail region.

In this paper we provide suggestions for the processes responsible for these plasma waves. The most interesting peculiarities of the wave activity around Mars is the sharp pcrease of wave intensity in the magnetosheath region. This increase is attributed to two different physical mechanisms. High frequency waves are excited at the shock front due to currents flowing along the front; these ion acoustic waves are corrected inside by the solar wind. The low frequency waves $(-100 \mathrm{~Hz})$ close to the inside boundary were, we betieve, generated by heavy Martian ions diffusing through the planetopause into the magnetosheath.

Introduction

Plasma wave activity in the neighborhood of the Martian bow shock were measured for the first time by an instrument package canied aboard the Soviet spacecraft Phobos. The phen wave system (PWS) carried out electric field measmements in a wide frequency range from dc to $150 \mathrm{kHz}$. The first results of these measurements have been published by Grad et al. [1989] and they showed the presence of significant wave activity, which varied in character as the spacecraft moved across different plasma regions. In this paper we provide suggestions for the processes responsible for these plasm waves in the neighborhood of the Martian bow shock and magnetosheath.

The observations discussed in this paper are restricted to the first elliptic orbit of the Phobos 2 spacecraft, which passed its perispsis of $867 \mathrm{~km}$ at 18.39 U.T. on February $1,1989$. More orbital details concerning this orbit can be found in Sagdeev and Zakharov [1989]. The results of the wave activity observed by the PWS on the dayside of the planet Mars are shown in Figure 1 and compared to the electron spectra, as measured by the HARP [Shutte et al., 1989], and to the magritude of the magnetic field [Riedler et al., 1989]. The main feames of the different plasma regions are also given in the figme caption, as well as later in the text. During this first encounter (1 Feb. 1989) the unperturbed solar wind parameters were $\mathrm{n}=2 \mathrm{~cm}^{-3}, \mathrm{u}=8 \times 10^{7} \mathrm{~cm} / \mathrm{s}, \mathrm{B}=(3-5) \mathrm{nT}$.

Copyright 1990 Dy the American Geophysical Union.

Puper number 90GL00929

$0094-8276 / 90 / 90 \mathrm{GL}-00929 \$ 03.00$
Precursor Region and the Shock-Foot

The electrostatic waves detected in the upstream region are typical for planetary bow shocks (e.g., similar waves were detected while Phobos crossed the Earth bow shock). These waves are probably electron Langmuir oscillations, excited in the solar wind by a beam instability driven by high-energy electrons, which are accelerated at the shock-foot and travel upstream along the magnetic field lines. The electron energy spectra, measured by the HARP instrument are shown in Shutte et al. [1989]. The bursts of high energy electrons are correlated with the bursts of electron plasma ascillations in the precursor region. The observed bandwidth of these waves is broadened, because of filter cross-overs with attenuations of 3 $\mathrm{dB}\left(\mathrm{f}_{\mathrm{pe}} \sim 14 \mathrm{kHz}\right)$

Another feature, usually observed upstream of planetary bow shocks, is the foot region. The outer boundary of the shock-foot is the envelope of the trajectories of solar wind ions reflected from the shock front and returned to the up-

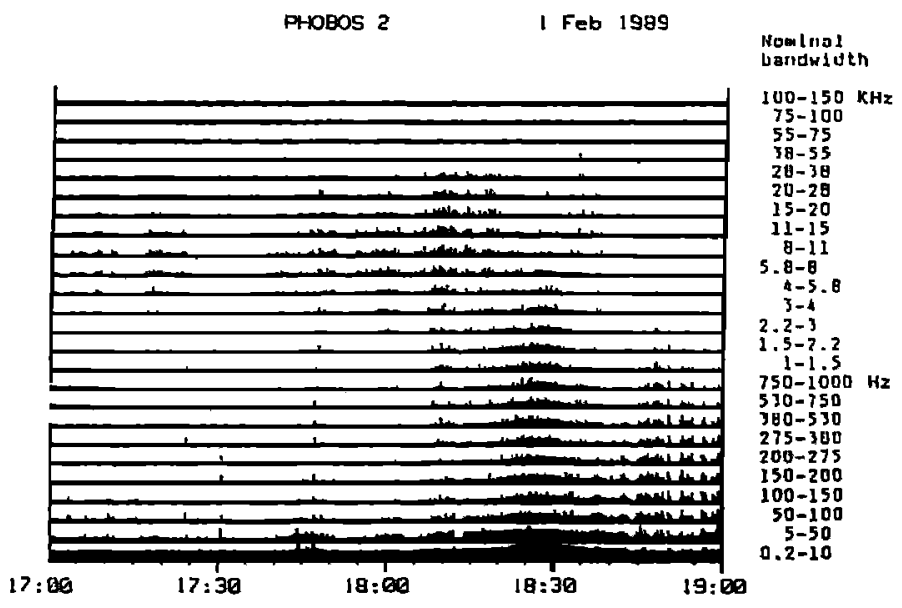

Figure 1. From the top the continuous data sequence of the total magnetic field [nT], the electron spectrum and wave intensity is exhibited as recorded by MAGMA, HARP and PWS during the first elliptic orbit, February 1, 1989. The wave intensity is given in the $-90,-40 \mathrm{dBV}$ range ( $-40 \mathrm{dBV}=$ $10 \mathrm{mV}$ r.m.s.); the time of the bow shock (18:25) and planetopause (18:37) crossing is also indicated. The spacecraft crossed the outer boundary of the shock foot at 18:08, 18:12 and 18:14. It is identified as a change in wave character, appearance of a tail in the electron distribution, and as the beginning of a continuous increase of the magnetic field. The shock ramp can be more easily identified by the change in electron density than from the broad band plasma waves. The planetopause which separates the shocked magnetosheath from the obstacle is clearly identifiable in all the dath. 
stream region after completing a partial gyration in the solar wind magnetic field. The shock-foot is characterized by a gradual increase of the plasma density and the magnetic field. The observed foot thickness was about $1.2 \div 1.710^{3} \mathrm{~km}$, comparable to the Larmor radius of reflected protons ( $r_{p}=$ $\mathrm{u} / \omega_{\mathrm{Hp}}=0.9 \div 1.810^{3} \mathrm{~km}$ ), typical for quasi perpendicular shocks [Sagdeev, 1964].

A gyrating beam of reflected protons in the solar wind gives rise to a plasma wave instability, propagating almost perpendicularly to the magnetic field, with a frequency in the vicinity of low-hybrid resonance, $\omega_{\mathrm{LH}}{ }^{2}=\omega_{\mathrm{He}} \omega_{\mathrm{Hi}}$. The dispersion relation for this instability is [Davidson et al., 1977]:

$$
\begin{gathered}
\mathrm{D}(\omega, \mathrm{k})=K \omega_{\mathrm{pe}}^{2} / \omega_{\mathrm{He}}^{2}- \\
-\left[\omega_{\mathrm{pe}}^{2} \mathrm{k}_{1}^{2} / \mathrm{k}^{2} K+\omega_{\mathrm{pp}}^{2}\right] / \omega^{2}=0 \\
\text { where } K=1+\omega_{\mathrm{pe}}^{2} / \mathrm{k}^{2} \mathrm{c}^{2}
\end{gathered}
$$

where $\omega_{\mathrm{pe}}, \omega_{\mathrm{pp}} \omega_{\mathrm{pi}}$ are the Langmuir frequencies of electrons, protons and heavy ions, respectively; the index $\mathrm{H}$ denotes the gyrofrequency for the same species; $k$ is the wave vector, $k_{\| l}$ is its component parallel to the magnetic field. For short wave length, $\mathrm{k}>\omega_{\mathrm{pe}} / \mathrm{c}$ the frequency of the waves is changing proportional to $k_{\|,}$, from $\omega_{\mathrm{LH}}$ to $\omega \simeq \omega_{\mathrm{He}} k_{\|} / \mathbf{k}$. In the case of long wavelength, $k<\omega_{\text {pe }} / c$, electromagnetic effects become important: and the whistler mode is excited. The growth rate of the instability described by Equation 1 is:

$$
\gamma=\mathrm{n}_{\mathrm{R}} / \mathrm{n}_{\mathrm{O}} \mathrm{v}_{\mathrm{A}}{ }^{2} /\left(\Delta \mathrm{u}^{2}\right) \omega_{\mathrm{LH}}{ }^{2} / \omega K
$$

where $v_{A}$ is the Alfven velocity, $n_{0}$ is the solar wind density and $n_{R}$ is the density and $\Delta u$ is the velocity spread of the reflected protons. The maximal growth rate corresponds to small $k_{\| l} \leq \sqrt{ }\left(\left(m_{e} / m_{p}\right)\left(k^{2}+\omega_{\left.\left.p e^{2} / c^{2}\right)\right)}\right.\right.$ when the frequency of the oscillations is close to the lower hybrid resonance. On the first elliptic orbit around Mars this frequency was of the order of $10 \mathrm{~Hz}$ at the shock-foot. According to Equation (1) $\mathbf{k}_{\sharp} \neq 0$, hence a finite electric field parallel to $B$ is present. This field leads to a stochastic acceleration along $B$ of those electrons which are in Landau resonance with these low-frequency waves, $\omega=\mathbf{k}_{\|} \mathbf{v}_{\|}$

This stochastic acceleration is described by the quasilinear diffusion equation from which it is easy to obtain the relation that connects the energy of the accelerated electrons, $E_{\mathrm{e}}$, with the electric field, $\mathrm{E}$, of the lower hybrid oscillation and the shock-foot thickness, $\mathrm{L}_{\mathrm{f}}$

$$
E_{e}{ }^{2} \simeq \mathrm{e}^{2} \mathrm{E}^{2} \mathrm{k}_{\|} \mathrm{L}_{f} / \mathrm{k}^{2}
$$

In quasi steady state the growth rate of the waves excited at the shock-foot by the reflected protons, must be compensated by the Landau damping of these waves due to their resonance absorption by electrons: $\left(n_{\mathrm{R}} / \mathrm{n}_{\mathrm{o}}\right)\left(\mathrm{v}_{\mathrm{A}}{ }^{2} /(\Delta \mathrm{u})^{2}\right)=\left(\mathrm{n}_{\mathrm{Te}} / \mathrm{n}_{0}\right)\left(\mathrm{m}_{\mathrm{e}} \mathrm{c}^{2} /\right.$ $\left.E_{\mathrm{e}}\right)\left(\omega_{\mathrm{He}} / \omega_{\mathrm{pe}}\right)^{2}$, where $\mathrm{n}_{\mathrm{Te}}$ is the density of the superthermal electrons. To obtain this estimate we have used the following expression for the Landau damping rate due to electrons

$$
\gamma_{e}=\pi /(\partial \mathrm{D} / \partial \omega) \omega_{\mathrm{pe}}^{2} / \mathrm{n}_{\mathrm{o}} \mathrm{k}^{2} \partial \mathrm{f}_{\mathrm{d}} / \partial \mathrm{v}_{\mathrm{N}}\left(\omega / \mathrm{k}_{\|}\right)
$$

$$
=\pi / 2 \omega_{\mathrm{He}}^{2} \omega / \mathrm{n}_{\mathrm{o}} \mathrm{k}^{2} K \quad \partial \mathrm{f}_{\mathrm{d}} / \partial \mathrm{v}_{\mathrm{\| l}}\left(\omega / \mathrm{k}_{\mathrm{ll}}\right)
$$

where $f_{e}$ is the electron distribution function over paralled velocities, and the approximations $\partial \mathrm{f}_{\mathrm{f}} \partial \mathrm{v}_{\mathrm{f}} \simeq \mathrm{n}_{\mathrm{Te}} \mathrm{m} / E_{\mathrm{e}}$ and $\omega_{\mathrm{pe}} / \mathrm{c} \mathrm{k} \sim$ were used. The second balance condition requires that the energy pumped into the waves by the proton bear must be compensated by the energy gain of the accelerated electrons: $\mathrm{n}_{\mathrm{R}} \mathrm{m}_{\mathrm{p}} \mathrm{u}^{2} \Delta \mathrm{u}=2^{1 / 2} \mathrm{n}_{\mathrm{Te}} E_{\mathrm{e}}^{3 / 2 / \mathrm{m}_{\mathrm{e}}} \mathrm{i}^{1 / 2}$.

These balance conditions yield the following relations for the energy $E_{\mathrm{e}}$ and density $\mathrm{n}_{\mathrm{Te}}$ of the accelerated electrons [Shapiro and Shevchenko, 1988]:

$$
\begin{aligned}
& E_{\mathrm{e}}=\mathrm{m}_{\mathrm{p}} \mathrm{u}^{2}\left(\mathrm{~m}_{\mathrm{e}} / \mathrm{m}_{\mathrm{p}}\right)^{1 / 5}(\Delta \mathrm{u} / \mathrm{u})^{6 / 5}, \\
& \mathrm{n}_{\mathrm{Te}} \simeq \mathrm{n}_{\mathrm{R}}\left(\mathrm{m}_{\mathrm{e}} / \mathrm{m}_{\mathrm{p}}\right)^{1 / 5}(\Delta \mathrm{u} / \mathrm{u})^{-4 / 5} .
\end{aligned}
$$

Combining these equations with Equation (3), the wave electric field can be estimated to be:

$$
\mathrm{E}^{2} / 4 \pi \simeq \mathrm{n}_{\mathrm{o}} \mathrm{m}_{\mathrm{p}} \mathrm{u}^{2}\left(\mathrm{uv}_{\mathrm{A}} / \mathrm{c}^{2}\right)\left(\mathrm{m}_{\mathrm{p}} / \mathrm{m}_{\mathrm{e}}\right)^{3 / 5}(\Delta \mathrm{u} / \mathrm{u})^{12 / 5}
$$

In the above relation the shock-foot thickness was approcimated by the proton gyroradius: $L_{f} \tilde{-} u / \omega_{\mathrm{Hp}}$. Finally, we can obtain from Equations (4) and (5) that $E_{\mathrm{e}}-200 \mathrm{eV}, \mathrm{E} \sim$ $10 \mathrm{mV} / \mathrm{m}$ for the plasma parameters of the first elliptical atit and $\Delta u / u=0.5$. It is easy to show that the wave intensity given by Eq. (5) is enough to create such velocity spread in the reflected proton beam at distances $L_{\mathrm{f}}$ via quasilinear diffrsion. For $n_{R} \sim 0.2 n_{0}$ the distance to build up instability $10 u / \gamma$ is comparable to the foot length. Values of $E_{e}$ and $E$ are in reasonable agreement with the results from the HARP and PWS measurements. The ratio of the wave energy, $W=$ $\mathrm{E}^{2} / 4 \pi\left(\omega_{\mathrm{pe}} / \omega_{\mathrm{He}}\right)^{2}$ to the energy of the solar wind flow is of the order of a few percent.

\section{The Bow-Shock and Magnetosheath Region}

According to the PWS measurements the bow shock, abog the Sun-Mars line, is located at about $1.9-2.2 \times 10^{3} \mathrm{~km}$ above the surface. Immediately downstream from the bow shoct very intense wave activity is observed in a broad frequency range from tens of $\mathrm{Hz}$ up to several $\mathrm{kHz}$ [Grard et al, 196\%)

In this region, usually called the magnetosheath (its thictness is less than $10^{3} \mathrm{~km}$ along the Sun-Mars line), the domnant plasma component is the shocked solar wind. The plasma density increases in the magnetosheath, the flow velocity, $u$, is $\sim 100 \mathrm{~km} / \mathrm{s}$ and the magnetic field strength increases from about 12 to $25 \mathrm{nT}$. It is likely that due to turbulent diffusion through the planetopause, cold, heavy Martian ions also penetrate into the magnetosheath and are responsible for the observed wave activity.

Generally, heavy ions are 'picked up' by electric and magnetic fields in the solar wind in distances of the onder of a fom Larmor radius. However $\mathrm{r}_{\mathbf{i}}=u / \omega_{\mathrm{Hi}}=1000 \mathrm{~km}$, which i comparable or even greater than the thickness of the magasersheath, therefore there is no space for pick-up in the sheath. Heavy Martian ions form a cold beam in the shodtad solar wind plasma moving with a velocity of $\sim 100 \mathrm{~km}$, which leads to instabilities. The dispersion relation in the 
froquency range $\omega_{\mathrm{Hp}}<<\omega<\omega_{\mathrm{He}}$ taking into account the heavy ion component differs from Eq. (1):

$$
D(\omega, k)-\frac{\omega_{p i}^{2}}{(\omega+k \cdot u)^{2}}=0
$$

where $\omega_{p}$ is the Langmuir frequency of heavy ions.

If the density of heavy ions is sufficiently small, $\alpha=n_{i}$ $m_{p} m_{p} m_{i} \ll 1$, the excited frequencies are given by Equation (6) and the growth rate of the resonant beam oscillations is opad to:

$$
\gamma=\omega\left\{\alpha \omega_{\mathrm{LH}}^{2}\left[\omega_{\mathrm{LH}}^{2}+\omega_{\mathrm{He}}^{2} \mathrm{k}_{\|}^{2} /\left(\mathrm{k}^{2}+\omega_{\mathrm{pe}}^{2} / \mathrm{c}^{2}\right)\right]^{-1}\right\}^{1 / 3}
$$

The characteristic length necessary to build up the instability is $\mathrm{L}$ - $10 \mathrm{u} / \mathrm{y}$ - $(100-200 \mathrm{~km})$, which is much less than the crosal magnetosheath thickness. The energy source of the instability in the shocked solar wind is quite large. The ampliwdes of the excited waves can be obtained from the energy conservation relation:

$$
\omega \frac{\partial \mathrm{D}}{\partial \omega} \frac{E^{2}}{8 \pi}=\frac{\omega_{\mathrm{pe}}^{2}}{\omega_{\mathrm{He}}^{2}} \frac{\mathrm{E}^{2}}{4 \pi}=\beta \mathrm{n}_{\mathrm{p}} \mathrm{m}_{\mathrm{p}} \frac{\overline{\overline{\mathrm{u}}}^{2}}{2}
$$

where $\beta$ is the fraction of the solar wind energy which is lost doe to the instability. The Doppler shifted wave frequency, as measured by the spacectaft is $\omega_{0}-\omega+\mathbf{k u} \omega \alpha^{1 / \beta} \sim \gamma$. For megnetosheath conditions, this frequency range corresponds vo the excitation of broad band noise in the lower part of the spectrum, $\mathrm{f}<100-300 \mathrm{~Hz}$. The amplitude of these waves, eximation (8), is equal to $10-20 \mathrm{mV} / \mathrm{m}$ for $\beta$ 1 , in fairly good agreement with the observations [Grard et li., 1989].

An explanation for the upper part of the observed frequency spectrom probably requires a different physical mechanism. These electrostatic ion-acoustic waves [Grand et al., 1989] are excrited at the shock front by the electron current responsible for the magnetic field jump at the bow shock.

These oscillations are convected by the solar wind from the bow shock into the magnetosheath region. The growth rate of this instability is [Galeev and Sagdeev, 1984]:

$$
\begin{gathered}
\gamma=\sqrt{ }(\pi / 8) \omega_{p p}\left[\left(k v_{d}-\omega\right) / k v_{e}\right] \\
k \pi_{D}\left(1+k^{2} r_{D}^{2}\right)^{3 / 2}
\end{gathered}
$$

where $I_{D}$ is the Debye length. It follows from Equation (9) the the electron drift velocity must exceed the phase velocity of the ion acoustic wave or the ion-sound velocity $c_{s}{ }^{2}=$ $T_{d} t_{p}$. The electron drift velocity is equal to $v_{D}=v_{A e}$ (c/ope) $(\Delta B / B) / \Delta x$ (where $\Delta B / B$ is the magnetic field jump at the shock front, $\Delta x$ is its width, $v_{A e}=c \omega_{H d} / \omega_{p e}$ is the Alfven velocity for electrons), therefore the condition for the instability to exist in the case of $\Delta B / B \sim 1$ has the form $\Delta x \leq c / \omega_{\text {pe }}$ $V_{A d} / c_{A}$ i.e., the instability exists even for sufficiently wide shocks $\Delta x=100 \mathrm{c} / \omega_{\mathrm{pe}}$. It should be noted that to excite the acoustic instability at the shock front the electron temperature must exceed significantly the proton temperature. Another instability that can also excite short wavelength oscillations of ion-acoustic type is the negative energy mode driven by cold beam of Martian ions. This instability can develop in the downstream region where protons are hot $\left(T_{p}\right.$ $>\mathrm{T}_{\mathrm{e}}$ ). Dissipation of this negative energy mode due to resonant interaction with protons leads to the instability. For both types of instability the maximum growth rate corresponds to small wave length of the order of Debye length $\mathrm{kr}_{\mathrm{D}}$ $\sim 1$. In that case the frequency, modified by Doppler shift due to relative motion of the solar wind plasma and the spacecraft, is $\omega=\mathbf{k u} \sim \omega_{\mathrm{pe}} \mathrm{u} / \mathrm{v}_{\mathrm{Te}}<1-2 \mathrm{kHz}$. These waves could therefore be associated with the high frequency component of the wave activity observed downstream in the magnetosheath.

Returning now to the low frequency oscillations ( $\mathrm{f}<100 \mathrm{~Hz}$ ) excited by the cold Martian ions, it should be emphasized that they are likely to be responsible for the main features in the magnetosheath, namely i) heating of the electrons, ii) turbulent diffusion of the Martian ions through the planetopause, iii) tailward ion escape by collective pick-up mechanism.

Electron heating is connected with the acceleration of electrons that are in Cherenkov resonance $\left(\omega=k_{\|} v_{\|}\right)$with the waves, as is the situation at the shock-foot. The characteristic energy of the electron tail, obtained from Equation (3), is found to be equal to $300 \mathrm{eV}$, as compared to $100 \mathrm{eV}$ in the foot region, because the wave activity is more intense in the magnetosheath. The anomalous diffusion of heavy ions into the magnetosheath region as well as tailward ion escape both can be aftributed to momentum exchange mechanism between the solar wind and the planetospheric ions due to the oscillations. In order to estimate the efficiency of such mechanism it is necessary to calculate the effective collision frequency characterizing the loss of the momentum by the solar wind protons. In the reference frame of the planetospheric ions, solar wind protons form a cold beam; the rate at which this beam loses momentum, while exciting oscillations, can be calculated in the quasilinear approximation. The result in a simplified form could be written as

$$
v_{\text {eff }} m_{p} n_{0} u \approx \Sigma \gamma_{k} k N_{k}
$$

where $N_{k}$ is the spectral density of plasmons, $\boldsymbol{y}_{\mathbf{k}}$ is the growth rate of the mode, $N_{k}=1 / \omega\left(\omega_{\mathrm{pe}} / \omega_{\mathrm{He}}\right)^{2}\left|\mathrm{E}_{\mathbf{k}}\right|^{2 / 8 \pi}$. The lefthand side of this equation gives the rate at which protons of the solar wind lose momentum due to the instability; the righthand side is the rate at which excited oscillations absorb momentum. Equation (10) yields the following estimate for the effective collision frequency:

$$
v_{\text {eff }}=\frac{\omega_{p e}^{2}}{\omega_{H e}^{2}} \sum_{k} \gamma_{k}\left|E_{k}\right|^{2} / 4 \pi n_{o} m_{p} u^{2}
$$

Excited oscillations lead to vibrations of heavy ions across the magnetic field with the characteristic velocity $v^{\prime} \sim \mathrm{eE} / \mathrm{m}_{\mathrm{i}} \omega$ and finally to transverse ion diffusion with a corresponding ion diffusion coefficient $\mathrm{D}=\left\langle\mathrm{v}^{2}\right\rangle / V_{\mathrm{eff}} \sim\left(\mathrm{m}_{\mathrm{p}} / \mathrm{m}_{\mathrm{i}}\right)^{2} \mathrm{u}^{2} / \gamma \sim$ $3 \times 10^{11}-10^{12} \mathrm{~cm}^{2} / \mathrm{s}$. The ion mean free path $1 \sim \mathrm{D} / \mathrm{v}_{\mathbf{i}} \sim$ $100-300 \mathrm{~km}$ is large enough to allow the ions to penetrate deeply into the magnetosheath. 
It was shown by Lundin et al. [1989] that assuming a complete transfer of solar wind momentum to the ionospheric ions it is possible to obtain on ion escape rate $\left(\sim 4 \times 10^{26}\right.$ ions/s), close to the observed value. We wanted to prove that such effective momentum transfer could be due to a viscous like interaction 1 tween the solar wind and the planetary ionosphere via oscillations. Assuming that the wave energy $\mathrm{W}=\left(\omega_{\mathrm{pe}} / \omega_{\mathrm{He}}\right)^{2} \mathrm{E}^{2} / 4 \pi$ is of the order of the solar wind energy $\mathrm{n}_{\mathrm{o}} \mathrm{m}_{\mathrm{p}} \mathrm{u}^{2} / 2$ it follows from equation (11) that the mean free path of the solar wind protons $\mathrm{u} / \mathrm{v}_{\mathrm{eff}} \sim 10^{3} \mathrm{~km}$ is sufficiently small. Excited waves are propagating mainly across the magnetic field and may cross the planetopause. Being absorbed by cold Martian ions they create a tailward moving ion flux.

\section{The Planetosphere}

Intense and discrete bursts of plasma waves are observed inside the planetosphere, which is a region dominated by heavy ions of planetary origin. The durations of these bursts were 0.5 - $2 \mathrm{~min}$, and their spatial extent was $100-400 \mathrm{~km}$ [Grard et al., 1989]. It is important to note that correlated pulses of highly energetic electrons and heavy ions are also observed in this region [Rosenbauer et al., 1989; Lundin et al., 1989].

If Mars has an intrinsic magnetic field, the wave bursts can be interpreted as whistlers trapped and propagating in magnetospheric wave guides. The possible mechanism for whistler wave excitation is the fan instability driven by the high energy electron tail [Shapiro and Shevchenko, 1968]. Anomalous Doppler resonance between electrons and waves with frequencies below the electron gyrofrequency leads to an instability characterized by the following growth rate:

$$
\gamma_{\mathrm{e}} \approx\left(\omega_{\mathrm{He}}{ }^{2} / \mathrm{kv}_{\mathrm{e}}\right)\left(\mathrm{n}_{\mathrm{Te}} / \mathrm{n}_{\mathrm{o}}\right) \sim(1 \div 3) 10^{-2} \omega_{\mathrm{He}}
$$

where $v_{e}$ and $n_{\mathrm{Te}}$ are the characteristic velocity and density of the accelerated electrons. The scale length of this instability is sufficiently small, $L_{\text {inst }} \sim 10 \mathrm{ve} / \gamma-300+1000 \mathrm{~km}$, to allow it to develop in the planetosphere. The whistler waves are guided by magnetospheric wave guides where the plasma density is low and which are parallel to the magnetic field lines. Wave bursts are observed when the spacecraft crosses these wave guides. The absorption of these low frequency waves $(\omega<<$ $\omega_{\mathrm{He}}$ ) can also lead to the acceleration of ionospheric ions. Cherenkov resonance $(\omega=k v)$ is possible for energetic ions. This acceleration is described by the usual quasilinear equation for unmagnetized ions from which the scaling law for the perpendicular energy can be easily obtained

$$
E_{\perp}=\left[\pi e^{2} E^{2} \overline{\bar{k}}^{-3} / m_{i} \omega^{2} L_{M} v_{\|}^{-1}\right]^{2 / 5}
$$

where $\mathrm{L}_{\mathrm{M}}$ is the length scale required for the acceleration to develop. Taking $\mathrm{L}_{\mathrm{M}} \sim 100 \mathrm{~km}$ and $\mathrm{E} \sim 10 \mathrm{mV} / \mathrm{m}$, one obtains an ion energy $E_{\perp} \sim 1 \mathrm{keV}$.

Several other mechanisms can also be responsible for ion acceleration, e.g., reconnection of magnetic field lines in the tail region. Our main purpose was to point out that whistler waves are also important.

\section{Conclusion}

We have analyzed various wave phenomena which were observed in different regions of the dayside magnetosphere of the planet Mars. Some of these instabilities are typical in solm wind planet interactions, e.g., those found in the shock-foot and the bow shock regions. Closer to Mars, the loading of the solar wind by Martian ions becomes impartant. This loading results in very intense wave activity downstream of the bow shock and in the magnetosheath. Additional plasm features observed in the magnetosheath and magnetosphere such as ion escape and fluxes of accelerated electrons also seem to be connected with the observed wave activity.

\section{References}

Davidson, R.C., et al., Effect of finite $\beta$ for lower hybrid instability, Phzs. EL, 20, 301, 1977.

Galeev, A.A., R.Z. Sagdeev, Current instabilities and anomalous resistivity of plasma, in Handbook of Plasma Physics (ed. M.N. Rosenbluth and R.Z. Sagdeev), v2, $271,1984$.

Grard, R., et al., First measurements of plasma waves nex Mars, Nature 341, 607, 1989.

Lundin, R., et al, First measurements of the ionospheric plasma escape from Mars, Nature, 341, 609, 1989.

Riedler, W., et al., Magnetic fields near Mars: First results, Nature, 341, 604, 1989.

Rosenbauer, $H$., et al., Ions of Martian origin and plasm sheet in the Martian magnetosphere: Initial results of the TAUS experiment, Nature 341, 612, 1989.

Sagdeev, R.Z., Collective processes and shocks in rarefield plasmas in: Problems of plasma theory, 4, 20, ed: MA. Leontovich, Atomizdat, Moscow, 1964.

Sagdeev, R.Z., and A.V. Zakharov, Brief history of the Phobos mission, Nature, 341, 581, 1989.

Shapiro, V.D., On the nonlinear theory of the interaction of the monoenergetic beams with plasma, Yh. Eksp. Theor. Phzs. 44, 613, 1963.

Shapiro, V.D., and V.I. Shevchenko, Astrophysics and Space Physics Reviews, 425, 1988.

Shapiro, V.D., and V.I. Shevchenko, Quasi linear theory of relaxation of an electron beam in a magnetoactive plasm, Zh. Eksp.Teor.Fiz, 54., 1187 (Sov. Phys. JETP, 27, 635), 1968.

Shutte, N.M, et al., Observation of electron and ion fluxxes in the vicinity of Mars with the HARP spectrometer, Names 341. 614, 1989.

R. Z. Sagdeev, V. D. Shapiro, V. L Shevchenko, and A. Zacharov, Space Reseanch Institute, USSR Academy of Sciences, ul. Profsoyuznaya 88/34, Moscow, USSR 117810.

P. Király and K. Szegö, Central Research Institute for Physics, H-1525 P.O. Box 49, Budapest 114, Hungary.

A. F. Nagy, Space Physics Research Laboratory, The

University of Michigan, Ann Arbor, Michigan 48109-2143.

R. J. L. Grard, Space Science Department, ESA/ESTBC,

Domeinweg, Noordwijk, The Netherlands 2200.

(Received November 13, 1989 ; revised March 30, 1990; accepted April 2, 1990) 